\title{
OPEN Quantitative accuracy of virtual non-contrast images derived from spectral detector computed tomography: an abdominal phantom study
}

Jasmin A. Holz ${ }^{1,2}$, Hatem Alkadhi' ${ }^{3}$, Kai R. Laukamp ${ }^{1}$, Simon Lennartz ${ }^{1}$, Carola Heneweer ${ }^{1}$, Michael Püsken ${ }^{1}$, Thorsten Persigehl ${ }^{1}$, David Maintz ${ }^{1} \&$ Nils Große Hokamp ${ }^{1 凶}$

Dual-energy CT allows for the reconstruction of virtual non-contrast (VNC) images. VNC images have the potential to replace true non-contrast scans in various clinical applications. This study investigated the quantitative accuracy of VNC attenuation images considering different parameters for acquisition and reconstruction. An abdomen phantom with 7 different tissue types (different combinations of 3 base materials and 5 iodine concentrations) was scanned using a spectral detector CT (SDCT). Different phantom sizes ( $S, M, L$ ), volume computed tomography dose indices (CTDIvol 10, 15, $20 \mathrm{mGy}$ ), kernel settings (soft, standard, sharp), and denoising levels (low, medium, high) were tested. Conventional and VNC images were reconstructed and analyzed based on regions of interest (ROI). Mean and standard deviation were recorded and differences in attenuation between corresponding base materials and VNC was calculated (VNCerror). Statistic analysis included ANOVA, Wilcoxon test and multivariate regression analysis. Overall, the $\mathrm{VNC}_{\text {error }}$ was $-1.4 \pm 6.1 \mathrm{HU}$. While radiation dose, kernel setting, and denoising level did not influence $\mathrm{VNC}_{\text {error }}$ significantly, phantom size, iodine content and base material had a significant effect (e.g. S vs. M: $-1.2 \pm 4.9 \mathrm{HU}$ vs. $-2.1 \pm 6.0 \mathrm{HU} ; 0.0 \mathrm{mg} / \mathrm{ml}$ vs. $5.0 \mathrm{mg} / \mathrm{ml}:-4.0 \pm 3.5 \mathrm{HU}$ vs. $5.1 \pm 5.0 \mathrm{HU}$ and 35 -HU-base vs. 54 -HU-base: $-3.5 \pm 4.4 \mathrm{HU}$ vs. $0.7 \pm 6.5$; all $p \leq 0.05$ ). The overall accuracy of VNC images from SDCT is high and independent from dose, kernel, and denoising settings; however, shows a dependency on patient size, base material, and iodine content; particularly the latter results in small, yet, noticeable differences in VNC attenuation.

Computed tomography $(\mathrm{CT})$ provides morphological images with a high spatial resolution. To overcome the intrinsic low soft tissue contrast of CT, iodinated contrast media is frequently administered, particularly in abdominal CT. Modern dual-energy CT's (DECT) allow for the reconstruction of virtual non-contrast (VNC) images, which have the potential to replace true non-contrast scans.

Conventional CT uses one polychromatic $\mathrm{x}$-ray source emitting a wide energy spectrum, typically covering a range from $40-120(-140) \mathrm{kVp}$. In conventional CT, a scintillation detector registers the global loss of intensity due to tissue attenuation. Hence, a global attenuation profile of the scanned object is obtained. Images reconstructed from this data are visualized using Hounsfield units (HU). As such a global attenuation profile in single energy CT cannot differentiate the influence of Photoelectric effect and Compton scattering, two different materials may exhibit similar HU values. To overcome this limitation of conventional CT, dual-energy CT can be used. In DECT, two different energy spectra are employed to obtain separate attenuation profiles for lower and higher energy photons. Technological approaches to DECT are either emission-based (dual-source CT (DSCT), fast $\mathrm{kV}_{\mathrm{p}}$-switching DECT or twin-beam DECT) or detector-based (dual-layer, spectral detector CT (SDCT) $)^{1-3}$.

\footnotetext{
${ }^{1}$ Faculty of Medicine and University Hospital Cologne, Institute for Diagnostic and Interventional Radiology, University of Cologne, Kerpener Strasse 62, 50937 Cologne, Germany. ${ }^{2}$ Department of Radiation Oncology, University Hospital Bonn, University of Bonn, Venusberg Campus 1, 53127 Bonn, Germany. ${ }^{3}$ Institute of Diagnostic and Interventional Radiology, University Hospital Zurich, University of Zurich, Rämistrasse 100, 8091 Zurich, CH, Switzerland. ${ }^{\varpi}$ email: nils.grosse-hokamp@uk-koeln.de
} 


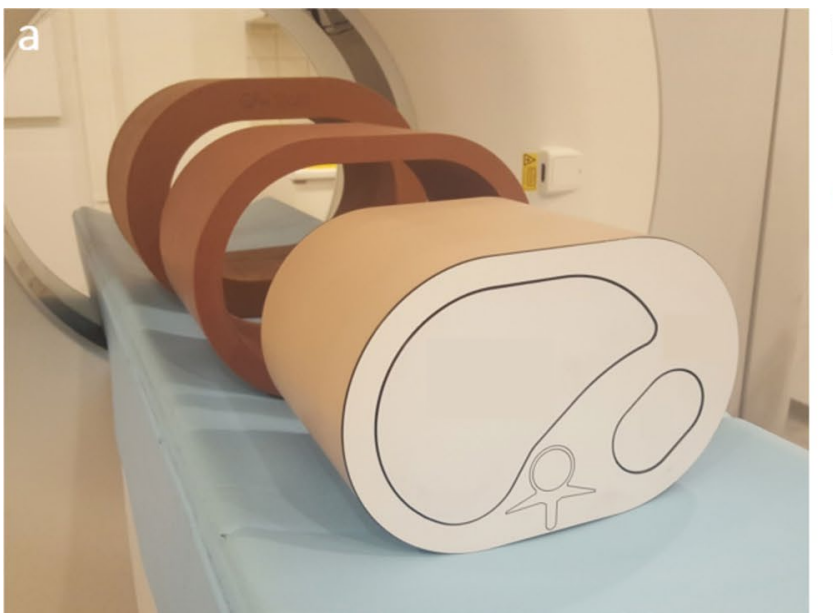

b
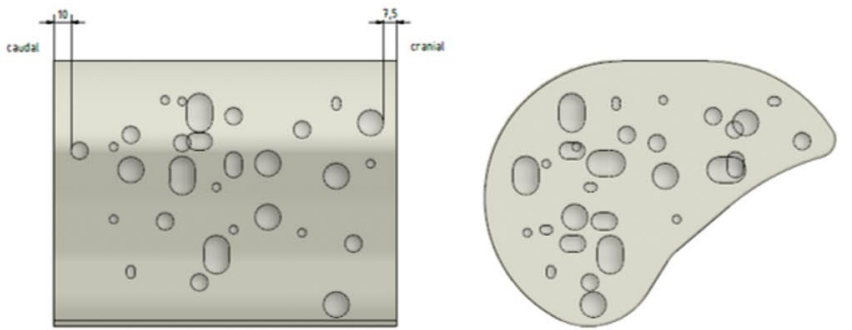

Figure 1. Anthropomorphic abdomen phantom with liver insert. (a) Photo of abdomen phantom size S and extension rings (employed to simulate sizes $\mathrm{M}$ and L). (b) Scheme of liver insert with lesion location and size in axial and sagittal plane.

\begin{tabular}{|l|l|l|l|l|l|l|l|}
\hline Tissue type & $\begin{array}{l}\text { Total attenuation } \\
(\mathbf{H U})\end{array}$ & $\begin{array}{l}\text { Base attenuation } \\
(\mathbf{H U})\end{array}$ & Iodine $(\mathbf{m g} / \mathbf{m l})$ & $\mathbf{1 0}(\mathbf{m m})$ & $\mathbf{1 5}(\mathbf{m m})$ & $\mathbf{1 0 \times 1 5}(\mathbf{m m})$ & $\mathbf{1 5 \times 2 2 . 5 ( \mathbf { m m } )}$ \\
\hline Matrix & 35 & 35 & 0.0 & - & - & - & - \\
\hline Parenchyma & 90 & 54 & 1.4 & - & - & - & - \\
\hline Lesion type 1 & 45 & 45 & 0.0 & 1 & 2 & - & 1 \\
\hline Lesion type 2 & 60 & 54 & 0.3 & 1 & - & - & 1 \\
\hline Lesion type 3 & 45 & 35 & 0.4 & 1 & - & 1 & 1 \\
\hline Lesion type 4 & 120 & 54 & 3.0 & 1 & 1 & 1 & 1 \\
\hline Lesion type 5 & 180 & 54 & 5.0 & 1 & 1 & 1 & 1 \\
\hline
\end{tabular}

Table 1. Specification of tissue types with liver lesions and size $>5 \mathrm{~mm}$. Of note, iodine concentrations as provided by the vendor are estimates only (as per phantom datasheet).

By separate assessment of low and high energy attenuation profiles, material decomposition becomes possible ${ }^{4-6}$. The most abundant applications of material decomposition comprise material specific maps for gout and iodine. In the latter, distribution of iodinated contrast media is visualized in quantitative maps ${ }^{4,7-11}$. These allow for the reconstruction of images that subtract the iodine-associated attenuation resulting in socalled virtual non-contrast (VNC) images ${ }^{12-20}$. While several studies demonstrated the general functionality of $\mathrm{VNC}^{21-26}$; studies which systematically investigating the impact of different patient-associated sizes, image acquisition and reconstruction parameters are sparse. Yet, it is essential to understand confounding factors for the evaluation of VNC performance ${ }^{8-11,27-30}$.

This study aims to comprehensively evaluate these parameters using an anthropomorphic abdomen phantom.

\section{Methods}

Phantom description. An anthropomorphic abdomen phantom with liver insert was used (QSA-453 and QSA-637, QRM GmbH, Moehrendorf, Germany) containing a total of 17 liver lesions with a size of $>5 \mathrm{~mm}$ (8 hyperdense and 9 hypodense lesions; Fig. 1).

Different patient sizes were simulated using no extension ring small $(\mathrm{S}, 300 \times 200 \mathrm{~mm})$, and with extension rings in size medium $(\mathrm{M}, 350 \times 250 \mathrm{~mm})$ and large $(\mathrm{L}, 400 \times 300 \mathrm{~mm})$. Matrix material of the phantom exhibited an attenuation of $35 \mathrm{HU}$ on conventional CT without iodine present. According to the vendor, the parenchyma's base material yields attenuation of $54 \mathrm{HU}$, which is increased by adding iodine to meet attenuation of $90 \mathrm{HU}$ on conventional images (Table 1). Base material and iodine amount of liver lesions varied to meet clinically encountered attenuation values of 45,60,120 and $180 \mathrm{HU}$ on conventional images (Table 1, Electronic supplement 1). In total, this resulted in 7 different types of tissue (5 lesion types + matrix + parenchyma, Table 1). Of note, iodine concentrations provided by the vendor were established using a DSCT; therefore, no accuracy calculations of iodine were conducted.

Data acquisition and image reconstruction. The phantom was placed in the isocenter of a spectral detector CT (SDCT, IQon, Philips Healthcare, Best, The Netherlands) and scanned with a slightly modified routine clinical abdomen protocol: tube voltage $120 \mathrm{kVp}$, fixed tube current-time product of $111 \mathrm{mAs}, 166 \mathrm{mAs}$, $222 \mathrm{mAs}$, resulting in volume computed tomography dose index $\mathrm{CTDI}_{\mathrm{vol}}$ of $10 \mathrm{mGy}, 15 \mathrm{mGy}$ and 20 mGy; field- 
of-view of $350 \mathrm{~mm}, 400 \mathrm{~mm}$ and $450 \mathrm{~mm}$ for phantom size S, M and L, respectively; collimation $64 \times 0.625 \mathrm{~mm}$; pitch 0.485 ; rotation time $0.33 \mathrm{~s}$; matrix $512 \times 512$.

Conventional images were reconstructed using the vendor's hybrid-iterative reconstruction algorithm (iDose ${ }^{4}$, Philips Healthcare, Best, The Netherlands). Virtual non-contrast images and iodine maps were reconstructed using a dedicated spectral reconstruction algorithm (Spectral, Philips Healthcare, Best, The Netherlands). As the reconstruction algorithm involves methods from iterative reconstruction, it allows for a choice of denoising level; here, no, medium, and strong denoising levels were chosen $(0 / 6,3 / 6 \text { and } 6 / 6 \text {, respectively })^{31}$. Furthermore, image definition (kernel) was varied between soft, standard, and sharp (A, B and C, Philips Healthcare, Best, The Netherlands). All images were reconstructed with a slice thickness of $2 \mathrm{~mm}$ with a section increment of $1 \mathrm{~mm}$ in axial plane (analogously to our institution's routine protocol). In total, the variation resulted in 81 reconstructions ( 3 patient sizes $\times 3$ radiation doses $\times 3$ denoising settings $\times 3$ kernels).

Data collection. Data was collected based on region of interest measurement (ROI). ROI were placed on conventional images, copied, and pasted to iodine maps and VNC images, respectively. ROI were placed in all 17 lesions (one ROI each), in the parenchyma (i.e., spleen insert) as well as the matrix material (two ROI each) and drawn as large as possible $(\mathrm{d} \geq 6 \mathrm{~mm})$. Attenuation on conventional and VNC images, as well as iodine concentration on iodine maps were recorded including corresponding standard deviations.

The $\mathrm{VNC}_{\text {error }}$ was calculated as difference between attenuation in $\mathrm{VNC}$ reconstruction $\left(\mathrm{HU}_{\mathrm{VNC}}\right)$ and the reported attenaution of the corresponding base material $\left(\mathrm{HU}_{\mathrm{base}}\right)$ :

$$
V N C_{\text {error }}=H U_{V N C}-H U_{\text {base }}
$$

Lesions with none, low, medium, and high iodine concentrations were grouped as indicated $(0.0 \mathrm{mg} /$ $\mathrm{ml}-0.3$ and $0.4 \mathrm{mg} / \mathrm{ml}-3.0$ and $5.0 \mathrm{mg} / \mathrm{ml}$, respectively).

Data analysis. All statistical analysis was performed using JMP Software (SAS Institute, Gary, USA). Continuous data is presented as mean \pm standard deviation (SD). Statistical analysis was carried out using one-way ANOVA or Wilcoxon signed-rank test with Tukey-Kramer and Steel-Dwass adjustment for multiple comparisons, respectively. Further multivariate regression analyis was used. Statistical significance was defined as $p \leq 0.05$. Waterfall diagrams were used to visualize $\mathrm{VNC}_{\text {error }}$ and its dependence on patient size, base material, and iodine content.

\section{Results}

Attenuation characteristics. Overall, attenuation ranged from 24.9-195.6 HU and 18.7-72.5 HU in conventional and VNC images, respectively. Iodine concentration ranged from 0.0 to $5.5 \mathrm{mg} / \mathrm{ml}$. Average $\mathrm{VNC}_{\text {error }}$ was $-1.4 \pm 6.1 \mathrm{HU}$, ranging from -16.3 to $18.5 \mathrm{HU}$. Detailed values for pooled analysis of all measurements are depicted in Table 2, example images are shown in Fig. 2.

VNC error and phantom size. No significant differences were found between small and large phantom settings $\left(\mathrm{VNC}_{\text {error }}:-1.2 \pm 4.9 \mathrm{HU}\right.$ and $-0.9 \pm 7.1 \mathrm{HU}$, respectively, $p \geq 0.05$, Fig. 3 ). Between small and medium as well as medium and large phantom settings, significant differences in $\mathrm{VNC}_{\text {error }}$ were observed $\left(\mathrm{VNC}_{\text {error }}\right.$ : $-1.2 \pm 4.9 \mathrm{HU},-2.1 \pm 6.0 \mathrm{HU}$ and $-0.9 \pm 7.1 \mathrm{HU}$, respectively, $p \leq 0.05$, Fig. 3).

VNC error and radiation dose. No significant differences were found between $10 \mathrm{mGy}, 15 \mathrm{mGy}$ and $20 \mathrm{mGy}$ acquisitions $\left(\mathrm{VNC}_{\text {error }}:-1.2 \pm 6.5 \mathrm{HU},-1.2 \pm 5.7 \mathrm{HU}\right.$ and $-1.7 \pm 6.1 \mathrm{HU}$, respectively, $p \geq 0.05$, Fig. 3).

VNC error and image definition (kernel). No significant differences were found between soft, standard or sharp image definition (kernel), $\left(\mathrm{VNC}_{\text {error }}:-1.4 \pm 6.1 \mathrm{HU}\right.$ for all kernels, $p \geq 0.05$, Fig. 3).

VNC error and denoising preset. No significant differences were found between no, medium and strong denoising, $\left(\mathrm{VNC}_{\text {error }}:-1.4 \pm 6.1 \mathrm{HU}\right.$ for all denoising levels, $p \geq 0.05$, Fig. 3).

VNC error and base material. Between all three base materials, significant differences were found (VNC $_{\text {error }}$ : 35-HU, 45-HU and 54-HU-base: $-3.5 \pm 4.4 \mathrm{HU},-5.0 \pm 3.9 \mathrm{HU}$ and $0.7 \pm 6.5 \mathrm{HU}$, respectively, $p \leq 0.001$, Fig. 3).

VNC error and iodine concentration. Between different iodine contents differences in $\mathrm{VNC}_{\text {error }}$ were observed $(0 \mathrm{mg} / \mathrm{ml}:-4.0 \pm 3.5 \mathrm{HU} ; 0.3 \mathrm{mg} / \mathrm{ml}:-6.2 \pm 4.0 \mathrm{HU} ; 0.4 \mathrm{mg} / \mathrm{ml}:-4.5 \pm 5.4 \mathrm{HU} ; 3.0 \mathrm{mg} / \mathrm{ml}$ : $-2.4 \pm 5.5 \mathrm{HU} ; 5.0 \mathrm{mg} / \mathrm{ml}: 5.1 \pm 5.0 \mathrm{HU} ; 1.4 \mathrm{mg} / \mathrm{ml}: 4.9 \pm 0.8 \mathrm{HU}$, all $p \leq 0.05$, except $0.0 \mathrm{mg} / \mathrm{ml}$ versus $0.3 \mathrm{mg} /$ ml: $p>0.05$; Fig. 3).

Regression analysis. In line with results from inter-group comparison, phantom size, base material, and iodine content were deemed significant parameters of $\mathrm{VNC}_{\text {error }}$ in regression analysis (all $p \leq 0.001$ ). Dose, kernel and denoising level, on the other hand, did not reach significance $(p=0.178, p=0.973$ and $p=0.879)$. In visual analysis, the impact of phantom size is not depictable in waterfall plots (Fig. 4), while it can be acknowledged that higher attenuation of base material as well as higher iodine content result in a positive $\mathrm{VNC}_{\text {error }}$. 


\begin{tabular}{|c|c|c|c|c|c|c|c|c|}
\hline \multirow[b]{2}{*}{ Tissue type } & \multicolumn{2}{|c|}{$\begin{array}{l}\text { Attenuation } \\
\text { (HU) }\end{array}$} & \multicolumn{2}{|c|}{ VNC (HU) } & \multicolumn{2}{|c|}{$\begin{array}{l}\text { Iodine }(\mathbf{m g} / \\
\mathrm{ml})\end{array}$} & \multicolumn{2}{|c|}{\begin{tabular}{|l|}
$\mathrm{VNC}_{\text {error }}$ \\
$(\mathrm{HU})$
\end{tabular}} \\
\hline & Mean & SD & Mean & SD & Mean & SD & Mean & SD \\
\hline Matrix & 30.3 & 2.4 & 33.0 & 1.1 & 0.0 & 0.0 & -2.0 & 1.1 \\
\hline Parenchyma & 96.5 & 1.5 & 58.9 & 0.8 & 1.4 & 0.1 & 4.9 & 0.8 \\
\hline Lesion type 1 & 46.6 & 3.2 & 40.0 & 3.9 & 0.2 & 0.1 & -5.0 & 3.9 \\
\hline Lesion type 2 & 58.7 & 3.8 & 47.8 & 4.0 & 0.4 & 0.2 & -6.2 & 4.0 \\
\hline Lesion type 3 & 43.8 & 4.8 & 30.5 & 5.4 & 0.5 & 0.2 & -4.5 & 5.4 \\
\hline Lesion type 4 & 117.3 & 4.9 & 51.6 & 5.5 & 2.6 & 0.3 & -2.4 & 5.5 \\
\hline Lesion type 5 & 181.8 & 5.8 & 59.1 & 5.0 & 4.8 & 0.3 & 5.1 & 5.0 \\
\hline
\end{tabular}

Table 2. Overall measurement results for attenuation, VNC, iodine and VNCerror for each tissue type.
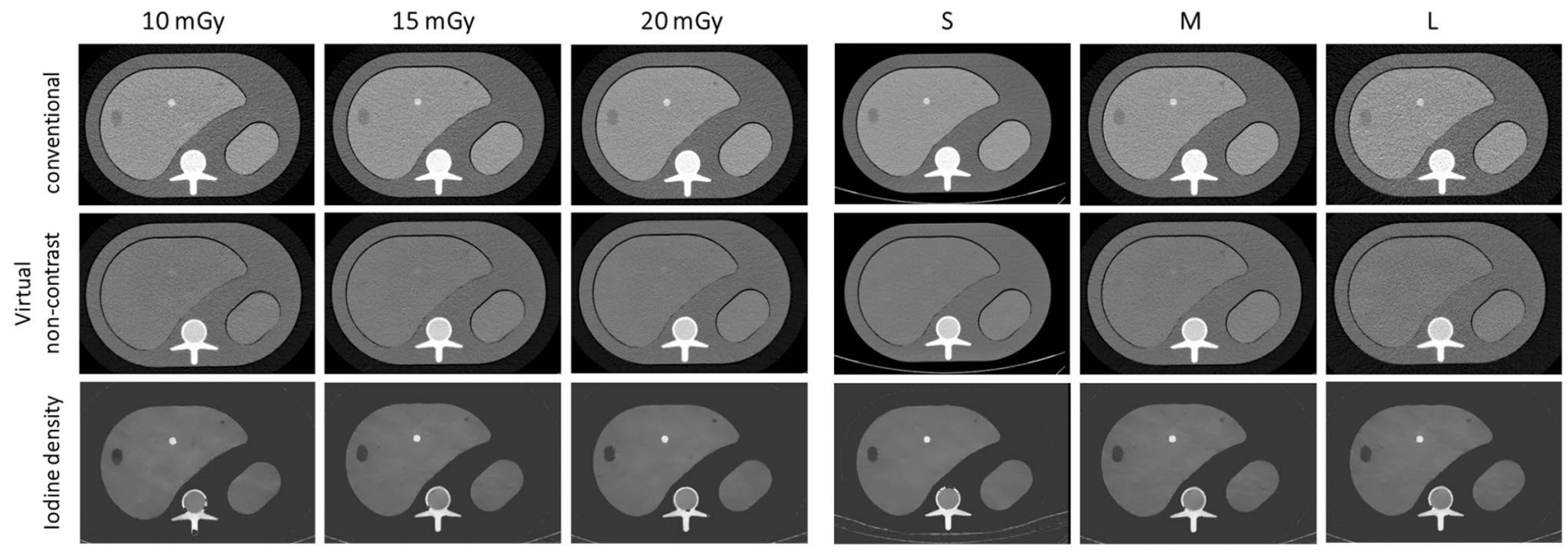

Figure 2. Example of axial slice images of liver lesions. Conventional (upper row), virtual non-contrast (middle row), and iodine density (lower row) images: LEFT) images of the medium sized phantom acquired with a CTDIvol of 10, 15, and 20 mGy are shown. RIGHT) images in different sizes (small, medium and large) are shown. Of note, conventional and VNC images are displayed with the institutional defaults for window level and width (WL 60, WW 360) and iodine (WL 2, WW 7), respectively. Images are cropped, hence the extension ring is not visible in S- and L-phantoms.

\section{Discussion}

This study systematically evaluated the influence of size, radiation dose, kernel, denoising level, base material, and iodine content on the accuracy of virtual non-contrast images using an anthropomorphic abdomen phantom. The key findings are that VNC performance is independent of radiation dose, kernel setting and denoising level, while patient size, base material and iodine concentration decrease performance slightly.

Overall, our data suggests a high accuracy of $\mathrm{VNC}$ with an overall $\mathrm{VNC}_{\text {error }}$ of $-1.4 \pm 6.1 \mathrm{HU}$. As per definition of $\mathrm{VNC}_{\text {error }}$, negative values indicate that the $\mathrm{VNC}$ attenuation values are lower compared to the expected attenuation of the base material; hence, that the iodine-associated attenuation has been slightly overestimated, a phenomenon known from earlier studies ${ }^{10}$. These findings are on the lower end of earlier reported VNC errors that range from -1.2 to $15 \mathrm{HU}^{17,28,30}$. This may be explained by the fact that in this study, unlike aforementioned investigations, a solid-state phantom was used. Here, the base materials exhibit attenuation different from water, whereas in most former studies aqueous dilutions of contrast media were used.

Interestingly, $\mathrm{VNC}_{\text {error }}$ was significantly lower in the medium-sized phantom as compared to small and large sizes. While the reason for this remains elusive, a possible hypothesis is that the medium sized phantom most closely resembles an average sized patient $(350 \times 250 \mathrm{~mm})$ potentially effecting model assumptions in image reconstruction ${ }^{2}$. Other studies ${ }^{28,30}$ reported that smaller phantoms yielded higher accuracy of VNC images. In line with that, we found lowest standard deviation with small size phantom.

In accordance with earlier reports we found that $\mathrm{VNC}_{\text {error }}$ is independent from kernel setting and denoising level ${ }^{9,27}$. Regarding radiation dose, which was deemed to not significantly impact $\mathrm{VNC}_{\text {error }}$ based on our data, there are opposing reports ${ }^{28,30}$ : While Van Hedent et al. report that lower radiation dose results in greater inaccuracy of VNC it needs to be acknowledge that their lowest dose ( $2 \mathrm{mGy}$ ) was markedly lower than the one used in this study $(10 \mathrm{mGy})^{28}$. Similarly, Si-Mohammed et al. employing CTDI $\mathrm{vol}_{\text {of }} 2.5 \mathrm{mGy}, 5 \mathrm{mGy}$ and $10 \mathrm{mGy}$, reported lower accuracy of $\mathrm{VNC}$ images $^{30}$. Of note, both studies used phantoms, which caused less attenuation compared to ours, enabling the use of such low doses.

The iodine content and the base material were deemed to significantly influence $\mathrm{VNC}_{\text {error }}$. In low concentrations, the iodine-associated attenuation is overestimated as indicated by a negative $\mathrm{VNC}_{\text {error }}$, while in 

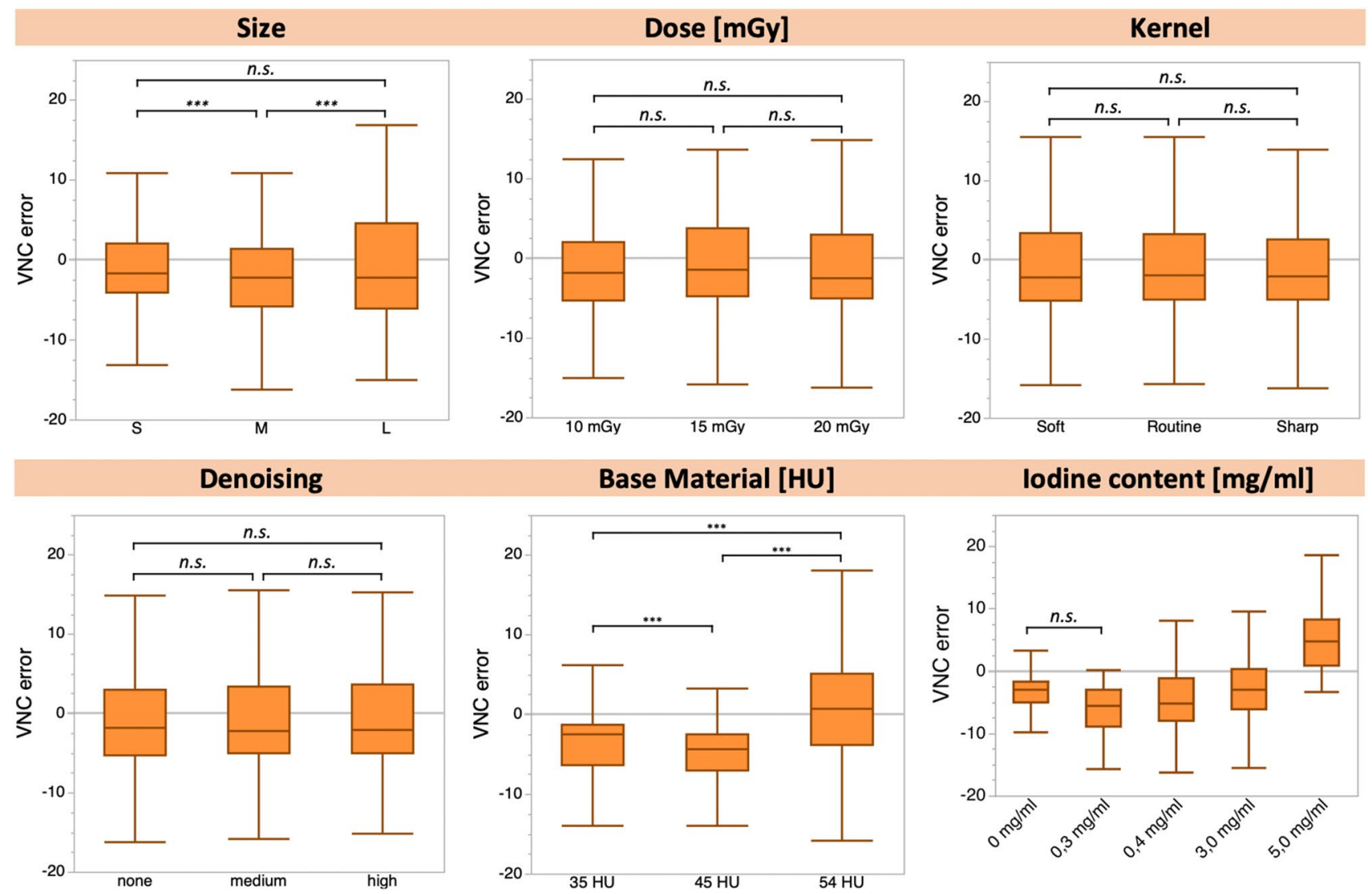

Figure 3. Box plots of the median $\mathrm{VNC}_{\text {error }}$ for phantom sizes, radiation doses, kernel settings, denoising levels, base materials, and iodine contents. Significant differences are indicated ${ }^{* * *} p<0.001$, n.s. $\left.p>0.05\right)$, except for iodine content (bottom right), for which all groups exhibit statistically significant $(p \leq 0.05)$ differences (unless indicated).

concentrations greater than $3 \mathrm{mg} / \mathrm{ml}$ an underestimation was found. Similar observation have been made by different groups ${ }^{4,29,30}$, e.g. Hua et al. also report a shift from under- towards overestimation between iodine contents of $2.5 \mathrm{mg} / \mathrm{ml}$ and $5 \mathrm{mg} / \mathrm{ml}^{4}$. Opposed to this, Van Hedent et al. reported no impact of iodine concentration on VNC performance ${ }^{28}$; however, the lowest concentration they used was $2 \mathrm{mg} / \mathrm{ml}^{28}$. Regarding the base materials, data with attenuation different from water is sparse. A study by Kim et al. investigated the influence of different solvents on iodine quantification accuracy ${ }^{11}$. Interestingly they found an overestimation in amino acid based solutions for concentrations lower than $1 \mathrm{mg} / \mathrm{ml}$, while an underestimation in higher concentrations ${ }^{11}$. They suggest that these differences might occur since in SDCT, two-material decomposition is performed for water and iodine. Hence, any additional material will tend to increase model uncertainty and therefore decrease performance ${ }^{4,11}$. This argumentation seems applicable to our findings as well. Further, it needs to be acknowledged, that a greater $\mathrm{VNC}_{\text {error }}$ can be expected in higher concentrations; however, we refrained from normalizing the error to ensure translation of our observations to clinical practice.

In general, our findings indicate a clinical applicability of VNC images as they show a reasonable accuracy under a variety of settings in an anthropomorphic phantom. Clinically, VNC reconstructions might be helpful in characterizing incidental findings, such as adenoma, or in increasing diagnostic confidence, e.g. in in hypodense lesions of the liver. For the latter, diagnosis is often found challenging in lesions $<1 \mathrm{~cm}$; here, a lack of iodine uptake is highly suggestive of a cystic origin and may increase confidence.

Yet, there are limitations that need to be discussed. First, we did not test extreme values for radiation dose or phantom size but focused on scan parameters typically encountered in abdominal imaging and average patients. Second, we did not evaluate accuracy of iodine reconstruction as there are numerous earlier reports for SDCT and other DECT-approaches ${ }^{8-11,27,28}$. It would also be of interest to investigate the dependency of $\mathrm{VNC}_{\text {error }}$ on different iodine background concentrations for the parenchyma and other organs. Further, true iodine concentrations for the used phantom were unavailable as the specifications provided by the vendor were validated using a dual source CT, only. No subjective image analysis was performed on the visibility of lesions; as VNC and iodine maps are usually consulted to obtain quantitative information whereas other spectral results are better suited for this purpose $\mathrm{e}^{23,32,33}$. The lesion size cut-off of $5 \mathrm{~mm}$ was chosen to ensure valid ROI placement. Assessment of actual performance for clinical decision making was out of scope of this study; hence, we do not advocate for the use of $\mathrm{VNC}$ instead of true non-contrast acquisitions if required a priori. Yet, our data together with earlier reports on clinical use-cases suggest that VNC closely resemble true non-contrast acquisitions irrespective of protocol settings and therefore may be used if deemed necessary after image acquisition. Another limitation is 

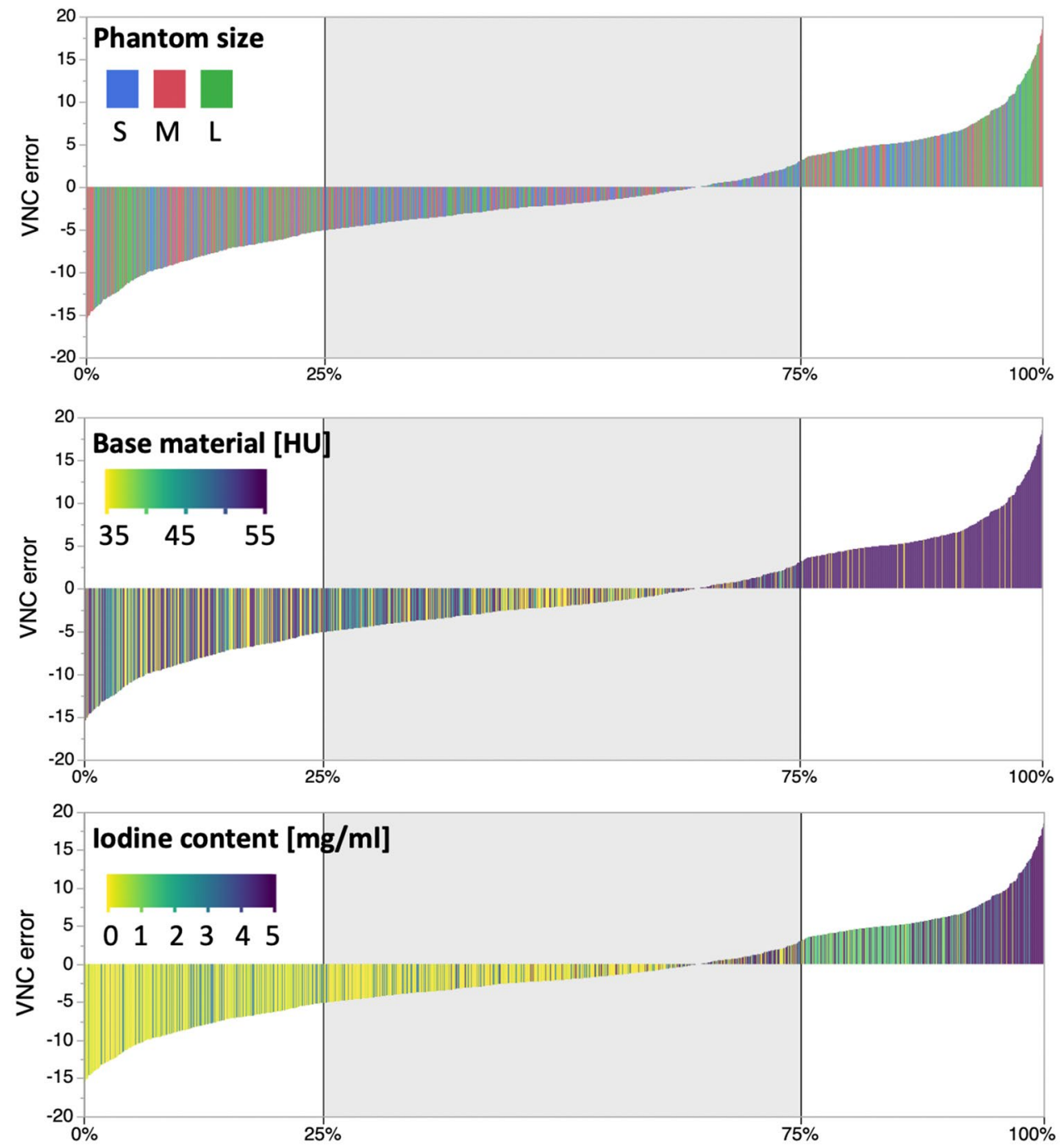

Figure 4. Waterfall diagrams visualize the all $\mathrm{VNC}_{\text {error }}$ sorted in ascending order and color-coded in dependency of phantom size, base material, and iodine content.

that we used a single CT scanner and interscan variation was not addressed in this study as those parameters were investigated in earlier studies (7).

In conclusion, the accuracy of virtual non-contrast reconstructions is independent from routinely applied dose ranges, kernel, and denoising level setting; however, we found a small dependency on patient size, base material, and iodine content.

Received: 18 June 2020; Accepted: 17 November 2020

Published online: 09 December 2020

\section{References}

1. McCollough, C. H., Leng, S., Yu, L. \& Fletcher, J. G. Dual- and multi-energy CT: principles, technical approaches, and clinical applications. Radiology 276, 637-653 (2015).

2. Große Hokamp, N. et al. Technical background of a novel detector-based approach to dual-energy computed tomography. Diagn. Interv. Radiol. 26, 68-71 (2020).

3. Patino, M. et al. Material separation using dual-energy CT: current and emerging applications. RadioGraphics 36, 1087-1105 (2016).

4. Hua, C. H., Shapira, N., Merchant, T. E., Klahr, P. \& Yagil, Y. Accuracy of electron density, effective atomic number, and iodine concentration determination with a dual-layer dual-energy computed tomography system. Med. Phys. 45, 2486-2497 (2018).

5. Mei, K. et al. Dual-layer spectral computed tomography: measuring relative electron density. Eur. Radiol. Exp. 2, 20 (2018).

6. Müller, F. C. et al. Optimising dual-energy CT scan parameters for virtual non-calcium imaging of the bone marrow: a phantom study. Eur. Radiol. Exp. 3(1), 46 (2019).

7. Große Hokamp, N. et al. Precision and reliability of liver iodine quantification from spectral detector CT: evidence from phantom and patient data. Eur. Radiol. 29, 2098-2106 (2019). 
8. Sellerer, T. et al. Dual-energy CT: a phantom comparison of different platforms for abdominal imaging. Eur. Radiol. https://doi. org/10.1007/s00330-017-5238-5 (2018).

9. Pelgrim, G. J. et al. Accuracy of iodine quantification using dual energy CT in latest generation dual source and dual layer CT. Eur. Radiol. 27, 3904-3912 (2017).

10. Jacobsen, M. C. et al. Intermanufacturer comparison of dual-energy CT iodine quantification and monochromatic attenuation: a phantom study. Radiology 287, 224-234 (2018).

11. Kim, H., Goo, J. M., Kang, C. K., Chae, K. J. \& Park, C. M. Comparison of iodine density measurement among dual-energy computed tomography scanners from 3 vendors. Invest. Radiol. 53, 321-327 (2018).

12. Leiva-Salinas, C. et al. Detection of parathyroid adenomas using a monophasic dual-energy computed tomography acquisition: diagnostic performance and potential radiation dose reduction. Neuroradiology 58, 1135-1141 (2016).

13. Lin, Y. M., Chiou, Y. Y., Wu, M. H., Huang, S. S. \& Shen, S. H. Attenuation values of renal parenchyma in virtual noncontrast images acquired from multiphase renal dual-energy CT: comparison with standard noncontrast CT. Eur. J. Radiol. 101, 103-110 (2018).

14. Sauter, A. P. et al. Dual-layer spectral computed tomography: virtual non-contrast in comparison to true non-contrast images. Eur. J. Radiol. https://doi.org/10.1016/j.ejrad.2018.05.007 (2018).

15. Lehti, L. et al. Reliability of virtual non-contrast computed tomography angiography: comparing it with the real deal. Acta Radiol. Open 7, 205846011879011 (2018).

16. Slebocki, K. et al. Incidental findings in abdominal dual-energy computed tomography. J. Comput. Assist. Tomogr. 41, 294-297 (2017).

17. Ananthakrishnan, L. et al. Spectral detector CT-derived virtual non-contrast images: comparison of attenuation values with unenhanced CT. Abdom. Radiol. 42, 702-709 (2017).

18. Laukamp, K. R. et al. Virtual non-contrast for evaluation of liver parenchyma and vessels: results from 25 patients using multiphase spectral-detector CT. Acta Radiol. https://doi.org/10.1177/0284185119893094 (2019).

19. Laukamp, K. R. et al. Evaluation of the liver with virtual non-contrast: single institution study in 149 patients undergoing TAVR planning. Br. J. Radiol. 93, 20190701 (2020).

20. Riederer, I. et al. Acute infarction after mechanical thrombectomy is better delineable in virtual non-contrast compared to conventional images using a dual-layer spectral CT. Sci. Rep. 8, 1-5 (2018).

21. Tawfik, A. M. et al. Comparison of dual-energy CT-derived iodine content and iodine overlay of normal, inflammatory and metastatic squamous cell carcinoma cervical lymph nodes. Eur. Radiol. 24, 574-580 (2014).

22. Apfaltrer, P. et al. Contrast-enhanced dual-energy CT of gastrointestinal stromal tumors. Invest. Radiol. 47, 65-70 (2012).

23. Große Hokamp, N. et al. Assessment of arterially hyper-enhancing liver lesions using virtual monoenergetic images from spectral detector CT: phantom and patient experience. Abdom. Radiol. 43, 2066-2074 (2018).

24. Faby, S. et al. Performance of today's dual energy CT and future multi energy CT in virtual non contrast imaging and in iodine quantification DECT technology. Med. Phys. 42, 4349-4366 (2015).

25. De Cecco, C. N. et al. Virtual unenhanced imaging of the liver with third-generation dual-source dual-energy CT and advanced modeled iterative reconstruction. Eur. J. Radiol. 85, 1257-1264 (2016).

26. Obmann, M. M. et al. Interscanner and intrascanner comparison of virtual unenhanced attenuation values derived from twin beam dual-energy and dual-source, dual-energy computed tomography. Invest. Radiol. 54, 1-6 (2019).

27. Sauter, A. P. et al. Accuracy of iodine quantification in dual-layer spectral CT: influence of iterative reconstruction, patient habitus and tube parameters. Eur. J. Radiol. 102, 83-88 (2018).

28. Van Hedent, S. et al. Impact of patient size and radiation dose on accuracy and precision of iodine quantification and virtual noncontrast values in dual-layer detector CT-a phantom study. Acad. Radiol. 27, 409-420 (2020).

29. Ehn, S. et al. Assessment of quantification accuracy and image quality of a full-body dual-layer spectral CT system. J. Appl. Clin. Med. Phys. https://doi.org/10.1002/acm2.12243 (2017).

30. Si-Mohamed, S. et al. Virtual versus true non-contrast dual-energy CT imaging for the diagnosis of aortic intramural hematoma. (2019).

31. Große Hokamp, N. et al. Virtual monoenergetic images from spectral detector CT as a surrogate for conventional CT images: unaltered attenuation characteristics with reduced image noise. Eur. J. Radiol. 117, 49-55 (2019).

32. Große Hokamp, N. et al. Improved visualization of hypodense liver lesions in virtual monoenergetic images from spectral detector CT: Proof of concept in a 3D-printed phantom and evaluation in 74 patients. Eur. J. Radiol. 109, 114-123 (2018).

33. Husarik, D. B. et al. Advanced virtual monoenergetic computed tomography of hyperattenuating and hypoattenuating liver lesions: ex-vivo and patient experience in various body sizes. Invest. Radiol. 50, 695-702 (2015).

\section{Author contributions}

J.H., H.A., D.M., and N.G.H. contributed to the conceptualisation and design. J.H., H.A., and N.G.H. contributed to the construction of the phantom, data acquisition and analysis. J.H., H.A., C.H., M.P., S.L., K.L., T.P. and N.G.H. contributed to the interpretation of data. All authors contributed to the draft or substantial revision of the manuscript. All authors have approved the submitted version (and any substantially modified version that involves the author's contribution to the study). All authors have agreed both to be personally accountable for the author's own contributions and to ensure that questions related to the accuracy or integrity of any part of the work, even ones in which the author was not personally involved, are appropriately investigated, resolved, and the resolution documented in the literature.

\section{Funding}

Open Access funding enabled and organized by Projekt DEAL. This work was partly funded through the Else Kröner-Fresenius-Stiftung (2018_EKMS.34 to Nils Große Hokamp).

\section{Competing interests}

SL: Received travel cost reimbursement and exemption from clinical duties for research outside this project as a part of a research agreement between Philips Healthcare and University Hospital Cologne. NGH: Receives research support from Philips Healthcare. NGH and DM: On the speakers bureau of Philips Healthcare. All other authors (JAH, HA, KRL, CH, MP, TP): Nothing to disclose.

\section{Additional information}

Supplementary information is available for this paper at https://doi.org/10.1038/s41598-020-78518-5.

Correspondence and requests for materials should be addressed to N.G.H. 
Reprints and permissions information is available at www.nature.com/reprints.

Publisher's note Springer Nature remains neutral with regard to jurisdictional claims in published maps and institutional affiliations.

(c) (i) Open Access This article is licensed under a Creative Commons Attribution 4.0 International License, which permits use, sharing, adaptation, distribution and reproduction in any medium or format, as long as you give appropriate credit to the original author(s) and the source, provide a link to the Creative Commons licence, and indicate if changes were made. The images or other third party material in this article are included in the article's Creative Commons licence, unless indicated otherwise in a credit line to the material. If material is not included in the article's Creative Commons licence and your intended use is not permitted by statutory regulation or exceeds the permitted use, you will need to obtain permission directly from the copyright holder. To view a copy of this licence, visit http://creativecommons.org/licenses/by/4.0/.

(C) The Author(s) 2020 